International Journal of Pure and Applied Mathematics

Volume 95 No. 4 2014, 553-566

ISSN: 1311-8080 (printed version); ISSN: 1314-3395 (on-line version)

url: http://www.ijpam.eu

doi: http://dx.doi.org/10.12732/ijpam.v95i4.8

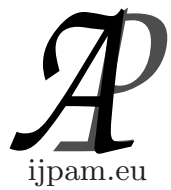

\title{
A NEW APPROACH TO SOLVE THE FRACTIONAL HARRY DYM EQUATION USING THE FRDTM
}

\author{
Mahmoud S. Rawashdeh \\ Department of Mathematics and Statistics \\ Jordan University of Science and Technology \\ P.O. Box 3030, 22110, Irbid, JORDAN
}

\begin{abstract}
In this research article, a new approach based on the Fractional Reduced Differential Transform Method (FRDTM) employed to solve the nonlinear fractional Harry Dym equation. In this work we present approximate analytical solutions of the time-fractional Harry Dym equation using the FRDTM and we find exact solution of Harry Dym equation using the RDTM. The fractional derivatives are described in the Caputo sense. The FRDTM doesn't require any discretization, linearization and therefore it reduces significantly the numerical computations compare with the existing methods such as the perturbation technique, differential transform method (DTM) and the Adomian decomposition method (ADM). Also, the results we obtained in this paper are in a good agreement with the exact solutions; hence, this technique is powerful and efficient as an alternative method for finding approximate and exact solutions for nonlinear PDEs.
\end{abstract}

AMS Subject Classification: 35J05, 35J10, 33E12, 33R11, 35A22

Key Words: reduced differential transform method, Caputo fractional derivative, fractional Harry Dym equation, fractional differential equation

\section{Introduction}

There are many physical applications in science and engineering can be rep-

Received: May 7, 2014

(c) 2014 Academic Publications, Ltd. url: www.acadpubl.eu 
resented by models using the fractional differential equations, which are quite useful for many physical problems. These equations are represented by linear and nonlinear PDEs and solving such fractional differential equations is very important. The Harry Dym equation is used in several physical applications and it was first introduced by Kruskal and Moser [17] and is credited to unpublished paper by Harry Dym in 1973-1974. Also, the Harry Dym equation is related to the Korteweg-de Vries equation ( $\mathrm{KdV}$ ) and it is also associated with the Sturm-Liouville operator. The Dym equation represents a system in which dispersion and non-linearity are coupled together. Harry Dym is a completely integrable nonlinear evolution equation and it is important because it obeys an infinite number of conservation laws; it does not possess the Painleve property.

Many authors applied numerical and analytic methods to solve linear and non-linear fractional differential equations. A few of these methods to name: The Differential Transform Method (DTM) [19] and [20], the Adomian Decomposition Method (ADM) [27] and [28], the Variational Iteration Method (VIM) [29], and the Homotopy Perturbation Method (HPM) [30]. The RDTM was first introduced by Y. Keskin in his Ph.D., [9], [10] and [11]. Also, M. Rawashdeh [22], [23], [24] and [25], used the RDTM to find approximate and exact solutions for many PDEs applications. The RDTM based on the use of the traditional DTM techniques. Usually, a few numbers of iterations needed of the series solution for numerical purposes with high accuracy. Recently, Keskin and Oturanc [11], used the FRDTM to solve fractional partial differential equations.

Moreover, S. Kumar, M.P. Tripathi and O.P. Singh [18], obtained approximate solution to the fractional Harry Dym equation. Finally, D. Kumar, J. Singh and A. Kiliman [13], used Sumudu transform method to solve the fractional Harry Dym equation.

In this paper, we consider the nonlinear fractional Harry Dym equation of the form

$$
D_{t}^{\alpha} U(x, t)=U^{3}(x, t) D_{x}^{3} U(x, t), \quad 0<\alpha \leq 1,
$$

subject to the initial condition

$$
U(x, 0)=\left(a-\frac{3 \sqrt{b}}{2} x\right)^{2 / 3},
$$

where $\alpha$ is a parameter describing the order of the fractional derivative and $U(x, t)$ is a function of $x$ and $t$. It is worth mentioning here we are using the fractional derivative in the Caputo sense. Note that when $\alpha=1$, equation (1.1) reduces to the nonlinear Harry Dym equation. 
Moreover, the exact solution of the Harry Dym equation is given by [15]:

$$
u(x, t)=\left(a-\frac{3 \sqrt{b}}{2}(x+b t)\right)^{2 / 3}
$$

where $a$ and $b$ are some constants.

The rest of this paper is organized as follows: In Section 2, we give some preliminaries and definitions of fractional calculus. In section 3, the fractional reduced differential transform method is introduced. Section 4 is devoted to apply the method to a test problem and present graphs to show the effectiveness of the FRDTM for some values of $x$ and $t$. In section 5 , we present an example in the special case $\alpha=1$. In section 6 , we present a table for example (4.1). Section 7 is discussion and conclusion of this paper.

\section{Basic Definitions of Fractional Calculus}

In this section, we give some of the main definitions and facts that we will use in our study. Some of these basic definitions are due to Liouville which are given as follow [2] and [3]:

Definition 2.1. A real function $f(x), x>0$ is said to be in the space $C_{\mu}, \mu \in \mathbb{R}$ if there exists a real number $q(>\mu)$, such that $f(x)=x^{q} g(x)$, where $g(x) \in C[0, \infty)$, and it is said to be in the space $C_{\mu}^{m}$ if $f^{(m)} \in C_{\mu}, m \in \mathbb{N}$.

Definition 2.2. For a function $f$, the Riemann-Liouville fractional integral operator of order $\alpha \geq 0$, is defined as:

$$
\left\{J^{\alpha} f(x)=\frac{1}{\Gamma(\alpha)} \int_{0}^{x}(x-t)^{\alpha-1} f(t) d t, \alpha>0, x>0 J^{0} f(x)=f(x)\right\} .
$$

Caputo and Mainardi [3], presented a modified fractional differentiation operator $D^{\alpha}$ in their work on the theory of viscoelasticity to overcome the disadvantages of the Riemann-Liouville derivative when someone tries to model real world problems.

Definition 2.3. The fractional derivative of $f$ in the Caputo sense can be defined as

$$
\left\{\begin{array}{l}
D^{\alpha} f(x)=J^{m-\alpha} D^{m} f(x)=\frac{1}{\Gamma(m-\alpha)} \int_{0}^{x}(x-t)^{m-\alpha-1} f^{(m)}(t) d t, \\
m-1<\alpha \leq m, m \in \mathbb{N}, x>0, f \in C_{-1}^{m}
\end{array}\right\} .
$$


Lemma 2.4. If $m-1<\alpha \leq m, m \in \mathbb{N}$ and $f \in C_{\mu}^{m}, \mu \geq-1$, then

$$
\left\{\begin{array}{l}
D^{\alpha} J^{\alpha} f(x)=f(x), x>0 \\
J^{\alpha} D^{\alpha} f(x)=f(x)-\sum_{k=0}^{m-1} f^{(k)}\left(0^{+}\right) \frac{x^{k}}{k !}, m-1<\alpha<m
\end{array}\right\} .
$$

It should be mentioned here, the Caputo fractional derivative is used because it allows traditional initial and boundary conditions to be included in the formulation of our problem.

\section{Analysis of the FRDTM}

In this section, we give the methodology of the FRDTM. So let's start with a function of two variables $u(x, t)$ which is analytic and $k$-times continuously differentiable with respect to time $t$ and space $x$ in the domain of our interest. Assume we can represent this function as a product of two single-variable functions $u(x, t)=f(x) \cdot g(t)$. From the definitions of the DTM, the function can be represented as follows:

$$
u(x, t)=\left(\sum_{i=0}^{\infty} F(i) x^{i}\right)\left(\sum_{j=0}^{\infty} G(j) t^{j}\right)=\sum_{k=0}^{\infty} U_{k}(x) t^{k},
$$

where $U(i, j)=F(i) \cdot G(j)$ is called the spectrum of $u(x, t)$. Some basic operations of the fractional reduced differential transformation can be obtained as follows [14] and [15]:

Definition 3.1. If $u(x, t)$ is analytic and continuously differentiable with respect to space variable $x$ and time $t$ in the domain of interest, then the $t$ dimensional spectrum function

$$
U_{k}(x)=\frac{1}{\Gamma(k \alpha+1)}\left[\frac{\partial^{\alpha k}}{\partial t^{\alpha k}} u(x, t)\right]_{t=t_{0}},
$$

is the reduced transformed function of $u(x, t)$, where $\alpha$ is a parameter which describes the order of time-fractional derivative. Throughout this paper, $u(x, t)$ represents the original function and $U_{k}(x)$ represents the reduced transformed function. The differential inverse transform of $U_{k}(x)$ is given by

$$
u(x, t)=\sum_{k=0}^{\infty} U_{k}(x)\left(t-t_{0}\right)^{k \alpha} .
$$


From equations (3.2) and (3.3) we can deduce

$$
u(x, t)=\sum_{k=0}^{\infty} \frac{1}{\Gamma(k \alpha+1)}\left[\frac{\partial^{\alpha k}}{\partial x^{\alpha k}} u(x, t)\right]_{t=t_{0}}\left(t-t_{0}\right)^{k \alpha} .
$$

Note that when $t=0$, Eq. (3.4) becomes

$$
u(x, t)=\sum_{k=0}^{\infty} \frac{1}{\Gamma(k \alpha+1)}\left[\frac{\partial^{\alpha k}}{\partial x^{\alpha k}} u(x, t)\right]_{t=0} t^{k \alpha} .
$$

Note that from the above discussion, one can realize that the FRDTM is derived from the power series expansion of a function. Some basic operations of the reduced differential transformation obtained from equations (3.1) and (3.2) are given in the table below:

\begin{tabular}{|l|l|}
\hline Functional Form & Transformed form \\
\hline$u(x, t)$ & $\frac{1}{\Gamma(k \alpha+1)}\left[\frac{\partial^{k \alpha}}{\partial t^{k \alpha}} u(x, t)\right]_{t=0}$ \\
\hline$\gamma u(x, t) \pm \beta v(x, t)$ & $\gamma U_{k}(x) \pm \beta V_{k}(x), \gamma$ and $\beta$ are constant. \\
\hline$u(x, t) \cdot v(x, t)$ & $\sum_{i=0}^{k} U_{i}(x) V_{k-i}(x)$ \\
\hline$u(x, t) \cdot v(x, t) \cdot w(x, t)$ & $\sum_{i=0}^{k} \sum_{j=0}^{i} U_{j}(x) V_{i-j}(x) W_{k-i}(x)$ \\
\hline$\frac{\partial^{n \alpha}}{\partial t^{n \alpha}} u(x, t)$ & $\frac{\Gamma(k \alpha+n \alpha+1)}{\Gamma(k \alpha+1)} U_{k+n}(x)$ \\
\hline$\frac{\partial^{n}}{\partial x^{n}} u(x, t)$ & $\frac{\partial^{n}}{\partial x^{n}} U_{k}(x)$ \\
$x^{m} t^{n} u(x, t)$ & $x^{m} U_{k-n}(x)$ \\
\hline$x^{m} t^{n}$ & $x^{m} \delta(k \alpha-n)$, where $\delta(k \alpha-n)=\left\{\begin{array}{ll}1, & \alpha k=n \\
0, & \alpha k \neq n\end{array}\right\}$ \\
\hline
\end{tabular}

Table 1: Basic operations of the FRDTM [9], [10] and [11]

Remark. In Table 1, $\Gamma$ represents the Gamma function, which is defined by:

$$
\Gamma(z):=\int_{0}^{\infty} e^{-t} t^{z-1} d t, \quad z \in \mathbb{C} .
$$

Notice that the Gamma function is the continuous extension to the fractional function. Throughout this paper, we will be using the recursive relation $\Gamma(z+$ $1)=z \Gamma(z), z>0$ to calculate the value of the Gamma function of all real numbers by knowing only the value of the Gamma function between 1 and 2 . 
Now, we illustrate the basic idea of the FRDTM by considering a general fractional nonlinear nonhomogeneous partial differential equation with initial condition of the form:

$$
D_{t}^{\alpha} U(x, t)+R(U(x, t))+N(U(x, t))=h(x, t),
$$

subject to the initial conditions

$$
U(x, 0)=f(x), U_{t}(x, 0)=g(x)
$$

where $D_{t}^{\alpha} U(x, t)$ is the Caputo fractional derivative of the function $U(x, t), R$ is the linear differential operator, $N$ represents the general nonlinear operator and $h(x, t)$ is the source term.

Applying the FRDTM to both sides of Eq. (3.7), we obtain:

$$
L(u(x, t))+R(u(x, t))+N(u(x, t))+F(u(x, t))=L(h(x, t)) .
$$

Using the FRDTM formulas in Table 1, we can find:

$$
L(U(x, t))=f(x)+u^{\alpha} L(g(x, t))-u^{\alpha} L(R(U(x, t))-N(U(x, t))) .
$$

Using the FRDTM inverse transform on both sides of (3.10) to get:

$$
U(x, t)=H(x, t)-L^{-1}\left(u^{\alpha} L(R(U(x, t))+N(U(x, t)))\right),
$$

where $H(x, t)$ represents the term coming from the source term and the prescribed initial conditions. Now from equation (3.8), we can write the initial condition as:

$$
U_{0}(x)=f(x) ; U_{1}(x)=g(x) .
$$

To find the remaining iterations, we first substitute equation (3.12) into equation (3.11) and then we find all the values of $U_{k}(x)$. Finally, we apply the inverse transformation to all the values $\left\{U_{k}(x)\right\}_{k=0}^{n}$ to obtain the approximate solution:

$$
\widehat{u}(x, t)=\sum_{k=0}^{n} U_{k}(x) t^{k \alpha},
$$

where $n$ is the number of iterations we need to find the intended approximate solution.

Hence, the exact solution of our problem is given by $u(x, t)=\lim _{n \rightarrow \infty} \widehat{u}(x, t)$. 


\section{The Fractional Harry Dym by the FRDTM}

In this section, we employ the FRDTM to the nonlinear time-fractional Harry Dym equation which is given by:

$$
D_{t}^{\alpha} U(x, t)=U^{3}(x, t) D_{x}^{3} U(x, t), \quad 0<\alpha \leq 1,
$$

subject to the initial conditions

$$
U(x, 0)=\left(a-\frac{3 \sqrt{b}}{2} x\right)^{2 / 3} .
$$

Applying the FRDTM to Eq. (4.1) and Eq. (4.2) we get:

$$
\begin{aligned}
& U_{k+1}(x) \\
& =\frac{\Gamma(k \alpha+\alpha)}{\Gamma(k \alpha+\alpha+1)}\left(\sum_{r=0}^{k} \sum_{s=0}^{r} \sum_{i=0}^{s} U_{i}(x) U_{s-i}(x) U_{r-s}(x) \frac{d^{3}}{d x^{3}} U_{k-r}(x)\right) .
\end{aligned}
$$

Note that,

$$
U_{0}(x)=\left(a-\frac{3 \sqrt{b} x}{2}\right)^{2 / 3} .
$$

Now, substitute Eq. (4.4) into Eq. (4.3) to obtain the following:

$$
\left\{\begin{aligned}
U_{1}(x) & =\frac{-\Gamma(\alpha) b^{3 / 2}}{\Gamma(\alpha+1)\left(a-\frac{3 \sqrt{b} x}{2}\right)^{1 / 3}}, \\
U_{2}(x) & =\frac{-\Gamma(\alpha) \Gamma(2 \alpha) b^{3}}{2 \Gamma(\alpha+1) \Gamma(2 \alpha+1)\left(a-\frac{3 \sqrt{b} x}{2}\right)^{4 / 3}}, \\
U_{3}(x) & =\frac{2^{4 / 3} b^{9 / 2} \Gamma(\alpha) \Gamma(3 \alpha)(15 \Gamma(\alpha) \Gamma(2 \alpha+1)-32 \Gamma(2 \alpha) \Gamma(\alpha+1))}{\Gamma(\alpha+1)^{2} \Gamma(2 \alpha+1) \Gamma(3 \alpha+1)(2 a-3 \sqrt{b} x)^{7 / 3}} .
\end{aligned}\right.
$$

We continue in this manner and after a few iterations, the differential inverse transform of $\left\{U_{k}(x)\right\}_{k=0}^{\infty}$ will provide us with the following approximate solution:

$$
\widehat{u}(x, t)=\sum_{k=0}^{\infty} U_{k}(x) t^{\alpha k}=U_{0}(x)+U_{1}(x) t^{\alpha}+U_{2}(x) t^{2 \alpha}+\ldots .
$$

Thus, the exact solution of the problem is given by $u(x, t)=\lim _{n \rightarrow 0} \widehat{u}_{n}(x, t)$. 
Hence, the approximate solution is convergent rapidly to the exact solution. Also, it only takes few terms to get analytic function. Now, we calculate numerical results of the approximate solution $u(x, t)$ for different values of $\alpha=0.25, \alpha=\frac{1}{3}, \alpha=0.5, \alpha=1$ and different values of $x$ and $t$.

The numerical results for the approximate solution obtained by FRDTM and the exact solution given by Mokhtari [15], are shown in figure 1 for constant values of $a=4$ and $b=1$ for different values of $x, t$ and $\alpha$.
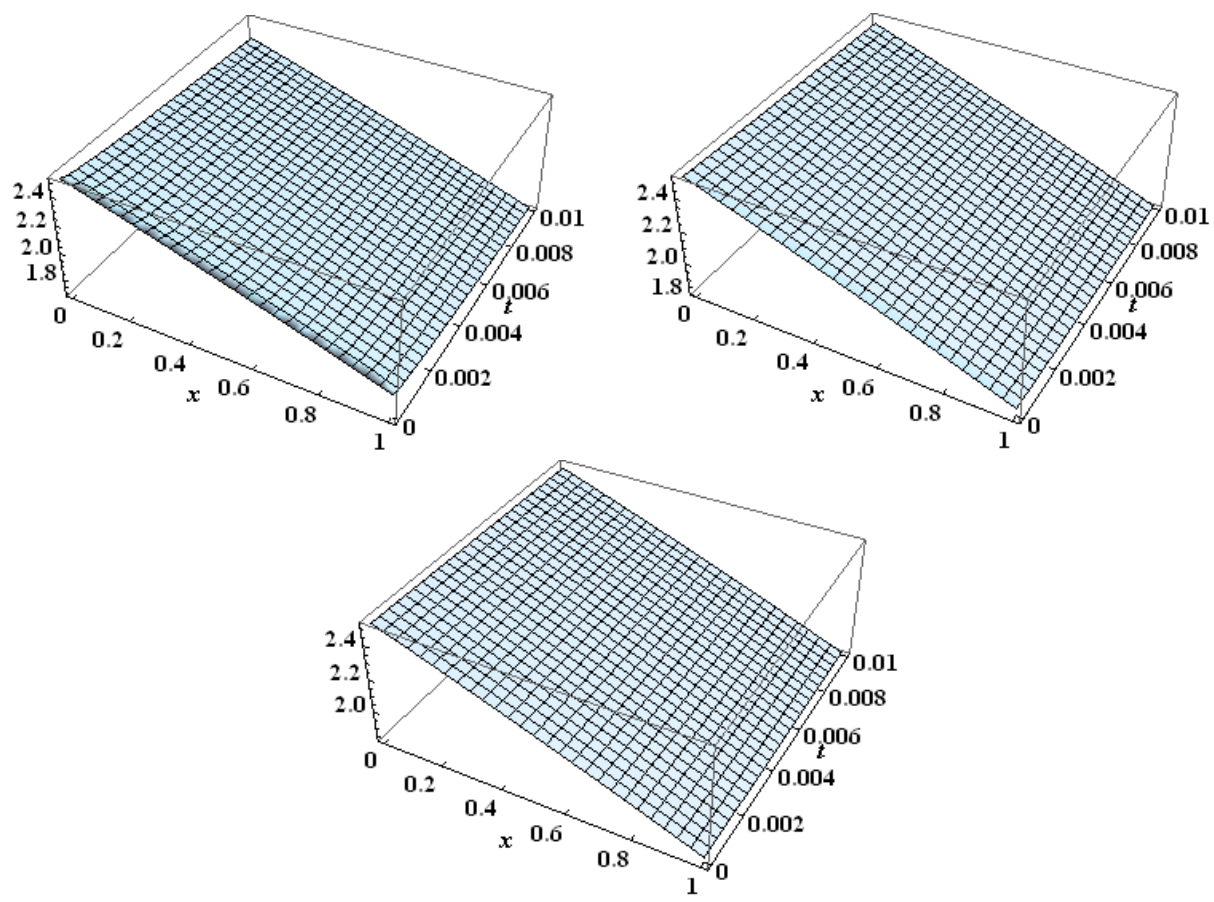

Figure 1: The behavior of $u(x, t)$ with respect to $x$ and $t$ with (a) $\alpha=0.25$; (b) $\alpha=\frac{1}{3}$; (c) $\alpha=0.25$

\section{Numerical Example}

In this section, we describe the method explained in section 2 by considering a numerical example to show the efficiency and the accuracy of the FRDTM. 


\subsection{Standard Harry Dym Equation}

Consider the nonlinear Harry Dym equation when $(a=4, b=1)$ given by:

$$
\frac{\partial}{\partial t} U(x, t)=U^{3}(x, t) \frac{\partial^{3}}{\partial x^{3}} U(x, t)
$$

subject to the initial condition

$$
U(x, 0)=\left(4-\frac{3}{2} x\right)^{2 / 3}
$$

Applying the FRDTM to Eq. (5.1) and Eq. (5.2) we get:

$$
U_{k+1}(x)=\frac{1}{k+1}\left(\sum_{r=0}^{k} \sum_{s=0}^{r} \sum_{i=0}^{s} U_{i}(x) U_{s-i}(x) U_{r-s}(x) \frac{d^{3}}{d x^{3}} U_{k-r}(x)\right),
$$

subject to the initial condition

$$
U_{0}(x)=\left(4-\frac{3 x}{2}\right)^{2 / 3}
$$

where the $U_{k}(x)$, is the transform function of the $t$-dimensional spectrum.

Now substitute Eq. (5.4) into Eq. (5.3) and for $k \geq 1$ we obtain

$$
\begin{gathered}
U_{1}(x)=-1\left(4-\frac{3 x}{2}\right)^{-1 / 3}, \quad U_{2}(x)=\frac{-1\left(4-\frac{3 x}{2}\right)^{-4 / 3}}{4}, \\
U_{3}(x)=\frac{-\left(4-\frac{3 x}{2}\right)^{-7 / 3}}{6},
\end{gathered}
$$

So after a few iterations, the differential inverse transform of $\left\{U_{k}(x)\right\}_{k=0}^{\infty}$ will give the following approximate solution:

$$
\widehat{u}(x, t)=\sum_{k=0}^{\infty} U_{k}(x) t^{k}=U_{0}(x)+U_{1}(x) t+U_{2}(x) t^{2}+U_{3}(x) t^{3}+\cdots
$$

Thus

$$
u(x, t)=\left(4-\frac{3 x}{2}\right)^{2 / 3}-\frac{t}{\left(4-\frac{3 x}{2}\right)^{1 / 3}}-\frac{t^{2}}{4\left(4-\frac{3 x}{2}\right)^{4 / 3}}-\frac{t^{3}}{6\left(4-\frac{3 x}{2}\right)^{7 / 3}}
$$




$$
\begin{array}{r}
-\frac{7 t^{4}}{48\left(4-\frac{3 x}{2}\right)^{10 / 3}}-\frac{7 t^{5}}{48\left(4-\frac{3 x}{2}\right)^{13 / 3}}-\frac{91 t^{6}}{576\left(4-\frac{3 x}{2}\right)^{16 / 3}}-\frac{13 t^{7}}{72\left(4-\frac{3 x}{2}\right)^{19 / 3}} \\
-\frac{2472^{1 / 3} t^{8}}{9(8-3 x)^{22 / 3}}-\frac{54342^{1 / 3} t^{9}}{81(8-3 x)^{25 / 3}}-\frac{135852^{1 / 3} t^{10}}{81(8-3 x)^{28 / 3}}+O[t]^{11} \\
=\left(4-\frac{3(t+x)}{2}\right)^{2 / 3} .
\end{array}
$$

This is the exact solution of the standard Harry Dym equation given in Eq. (5.1).

The numerical results for the exact, approximate and absolute errror obtained by FRDTM given by Mokhtari [15], are shown in figure 2 for constant values of $a=4$ and $b=1$ for different values of $x, t$ and $\alpha=1$.
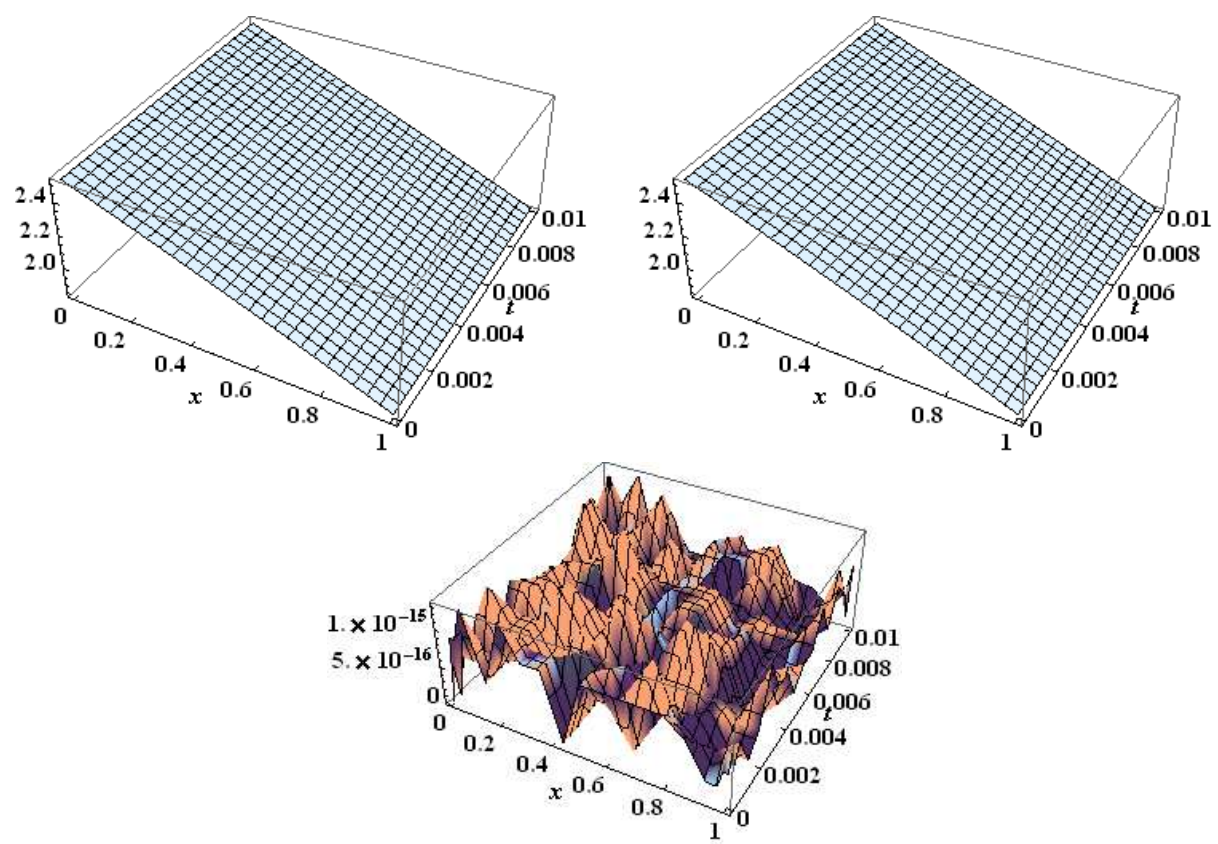

Figure 2: The exact, approximate and absolute error, respectively for example $5.1(\alpha=1, a=4, b=1)$ when $0<x<1$ and $0<t<0.01$ 


\section{Tables of Numerical Calculations}

The comparison of the results of the FRDTM and the exact solution for $\alpha=1$ is given in table 2 for different values of $x$ and $t$. We present in table 2 the results obtained by the FRDTM for different values of $x, t$ and $\alpha$ with only the $6^{\text {th }}$ order approximate solution $u(x, t)$ and the exact solution given in Eq. (4.1).

\begin{tabular}{|c|c|c|c|c|c|}
\hline \multirow[t]{2}{*}{$x$} & \multirow[t]{2}{*}{$t$} & \multirow{2}{*}{$\frac{\alpha=0.25}{\text { Numerical }}$} & \multirow{2}{*}{$\frac{\alpha=0.5}{\text { Numerical }}$} & \multicolumn{2}{|c|}{$\alpha=1$} \\
\hline & & & & Numerical & Exact \\
\hline \multirow[t]{4}{*}{0} & 0.002 & 1.95389 & 2.46318 & 2.51858 & 2.51858 \\
\hline & 0.004 & 1.83767 & 2.43951 & 2.51732 & 2.51732 \\
\hline & 0.006 & 1.75768 & 2.42128 & 2.51606 & 2.51606 \\
\hline & 0.008 & 1.69455 & 2.40585 & 2.5148 & 2.5148 \\
\hline \multirow[t]{4}{*}{0.3} & 0.002 & 1.73291 & 2.26811 & 2.26811 & 2.32581 \\
\hline & 0.004 & 1.60916 & 2.24345 & 2.3245 & 2.3245 \\
\hline & 0.006 & 1.52351 & 2.22443 & 2.32319 & 2.32319 \\
\hline & 0.008 & 1.45563 & 2.20833 & 2.32187 & 2.32187 \\
\hline \multirow[t]{4}{*}{0.6} & .002 & 1.49682 & 2.06426 & 2.12468 & 2.12468 \\
\hline & 0.004 & 1.36313 & 2.03839 & 2.12331 & 2.12331 \\
\hline & 0.006 & 1.26987 & 2.01843 & 2.12194 & 2.12194 \\
\hline & 0.008 & 1.19547 & 2.00153 & 2.12056 & 2.12056 \\
\hline \multirow[t]{4}{*}{1} & 0.002 & 1.14899 & 1.77551 & 1.84054 & 1.84054 \\
\hline & 0.004 & 0.995157 & 1.7476 & 1.83907 & 1.83907 \\
\hline & 0.006 & 0.885795 & 1.72602 & 1.83759 & 1.83759 \\
\hline & 0.008 & 0.797193 & 1.70773 & 1.83612 & 1.83612 \\
\hline
\end{tabular}

Table 2: The results obtained by the FRDTM for different values of $x$, $t$ and $\alpha$ for Example 4.1

\section{Conclusion}

In this paper, we applied the FRDTM to find approximate solutions of the nonlinear fractional Harry Dym equation for different values of $\alpha$ and the results we obtained in example (4.1) and example (5.1) were in excellent agreement with the exact solution. The FRDTM introduces a significant improvement in the fields over existing techniques because it takes less calculations and the 
number of iteration is less compared by other methods. My goal in the future is to apply the FRDTM to other nonlinear fractional PDEs which arises in other areas of science such as; Physics, Biology, Medicine and Engineering. Computations of the paper have been carried out using the computer package of Mathematica 7.

\section{Acknowledgements}

The author would like to express his appreciation and gratitude to the Editor and the anonymous referees for their comments and suggestions on this paper.

\section{References}

[1] R. Abazari, M. Abazari, Numerical simulation of generalized HirotaSatsuma coupled KdV equation by RDTM and comparison with DTM, Commun. Nonlinear Sci. Numer. Simulat., 17, (2012), 619-629.

[2] M. Caputo, Elasticitae dissipazione Zanichelli: Bologna, 1969.

[3] M. Caputo, Mainardi, F. linear models of dissipation in anelastic solids, Rivista del Nuovo Cimento, 1 (1971), 161-196.

[4] R. Cascaval, E. Eckstein, L. Frota, J. Goldstein, Fractional telegraph equations, Journal of Mathematical Analysis and Applications, 276, (2002), $145-159$.

[5] M. Garg, P. Manohar, Numerical solution of fractional diffusion-wave equation with two space variables by matrix method, Fractional Calculus and Applied Analysis, 13(2), (2010), 191-207.

[6] M. Garg, A. Sharma, Solution of space-time fractional telegraph equation by Adomian decomposition method, Journal of Inequalities and Special Functions, Volume(2) Issue(1), (2011), 1-7.

[7] H. He, Approximate analytical solution for seepage flow with fractional derivatives in porous media Computational Methods, Applied Mechanics and Engineering, 167, (1998), 57-68.

[8] R. Hilfer, Applications of Fractional Calculus in Physics, Singapore: World Scientific, 2000. 
[9] Y. Keskin, G. Oturan, Reduced Differential Transform Method for Partial Differential Equations, International Journal of Nonlinear Sciences and Numerical Simulation. 10(6)(2009)741-749.

[10] Y. Keskin, G. Oturan, Reduced Differential Transform Method for fractional partial differential equations, Nonlinear Science Letters A, 1(2), (2010), 61-72.

[11] Y. Keskin, G. Oturan, The reduced differential transform method: a new approach to fractional partial differential equations, Nonlinear Science Letters A, 1, (2010), 207-217.

[12] A.A. Kilbas, H.H. Srivastava, J.J. Trujillo, Theory and Applications of Fractional Differential Equations, Amsterdam: Elsevier, 2006.

[13] D. Kumar, J. Singh, A. Kiliman, An Efficient Approach for Fractional Harry Dym Equation by Using Sumudu Transform, Abstract and Applied Analysis Article.,ID 608943 (2013), 8 pages.

[14] K.S. Miller, B. Ross, An Introduction to the Fractional Calculus and Differential Equations, New York: John Wiley, 1993.

[15] R. Mokhtari, Exact solutions of the Harry-Dym equation, Communications in Theoretical Physics, vol. 55, No. 2, (2011), 204-208.

[16] S. Momani, Z. Odibat, V.S. Erturk, Generalized differential transform method for solving a space and time fractional diffusion-wave equation, Physics Letters A, 370, (2007), 379-387.

[17] M.D. Kruskal, J. Moser, Dynamical systems theory and applications lecturer notes physics, Berlin: Springer, 1975.

[18] S. Kumar, M.P. Tripathi, O.P. Singh, A fractional model of Harry Dym equation and its approximate solution, Ain Shams Engineering Journal. 4, (2013), 111-115.

[19] S. Momani, Z. Odibat, A generalized differential transform method for linear partial differential equations of fractional order, Applied Mathematics Letters, 21, (2008), 194-199.

[20] Z.Odibat, S. Momani, V.S. Erturk, Generalized differential transform method: Application to differential equations of fractional order, Applied Mathematics and Computation. 197, (2008) 467-477. 
[21] I. Podlubny, Fractional Differential Equations, San Diego: Academic Press, 1999.

[22] M. Rawashdeh, Improved Approximate Solutions for Nonlinear Evolutions Equations in Mathematical Physics Using the RDTM, Journal of Applied Mathematics and Bioinformatics, 3 No. 2, (2013), 1-14.

[23] M. Rawashdeh, Using the Reduced Differential Transform Method to Solve Nonlinear PDEs Arises in Biology and Physics, World Applied Sciences Journal, 23, No. 8, (2013), 1037-1043.

[24] M. Rawashdeh, N. Obeidat, On Finding Exact and Approximate Solutions to Some PDEs Using the Reduced Differential Transform Method, Applied Mathematics and Information Sciences, 8, No. 5, (2014), 1-6.

[25] M. Rawashdeh, Approximate Solutions for Coupled Systems of Nonlinear PDES Using the Reduced Differential Transform Method, Mathematical and Computational Applications; An International Journal, 19, No. 2, (2014), 161-171.

[26] M. Rawashdeh, Shehu Maitama, Solving Coupled System of Nonlinear PDEs Using the Natural Decomposition Method, International Journal of Pure and Applied Mathematics, Vol. 92, No. 5, (2014), 757-776.

[27] S.S. Ray, R.K. Bera, Solution of an extraordinary differential equation by Adomian decomposition method, J. Appl. Math., 4, (2004), 331-338.

[28] S.S. Ray, R.K. Bera, An approximate solution of a nonlinear fractional differential equation by Adomian decomposition method, Applied Mathematics and Computation, 167, (2005), 561-571.

[29] A. Sevimlican, An Approximation to Solution of Space and Time Fractional Telegraph equations by He's Variational Iteration Method, Mathematical Problems in Engineering, Volume 2010, Article ID 290631, 10 pages, doi:10.1155/2010/290631, 2010.

[30] A. Yildirim, He's homtopy perturbation method for solving the space and time fractional telegraph equations, International Journal of Computer Mathematics, 87(13), (2010), 2998-3006. 